\title{
GRAPHS WITH 1-FACTORS
}

\author{
DAVID P. SUMNER
}

\begin{abstract}
In this paper it is shown that if $G$ is a connected graph of order $2 n(n>1)$ not containing a 1-factor, then for each $k$, $1<k \leqq n$, there exists an induced; connected subgraph of order $2 k$ which also fails to possess a 1-factor. Several other sufficient conditions for a graph to contain a 1-factor are presented. In particular, it is seen that the connected even order line graphs and total graphs always contain a 1 -factor.
\end{abstract}

In this paper all graphs will be finite, undirected and without loops or multiple edges. A 1-factor of a graph is a spanning subgraph which is regular of degree one. Tutte [5] has characterized graphs which contain 1-factors. Beineke and Plummer [1] proved that every block with a 1factor always contains at least one more, and a result due to Petersen [4] showed that every cubic graph with no bridges contains a 1-factor.

Our purpose in this paper is to show (Theorem 2) that for any connected graph of order $2 n$ which does not contain a 1-factor and for each integer $k$, $1<k \leqq n$, there is an induced, connected subgraph of order $2 k$ which also fails to possess a 1-factor. Moreover, several other sufficient conditions for a graph to contain a 1 -factor are given. In particular, we see that the connected, even order line graphs and total graphs must always contain a 1 -factor.

Our terminology will conform to that in Harary [3].

Definition. If $x$ is an endpoint of the graph $G$ and $x$ is adjacent to $z$, then we will say that $z$ is the joint of $x$. If $x$ and $y$ are two endpoints of $G$ which have the same joint, then we will call $x$ and $y$ coincident endpoints.

We note that no graph with coincident endpoints can possess a 1-factor.

LEMMA 1. If $G$ is a connected, nontrivial graph with no coincident endpoints, then there exist adjacent points $x, y \in G$ such that $G-\{x, y\}$ is connected.

Proof. Since the lemma clearly holds for complete graphs, we assume that $G$ has diameter $d \geqq 2$. Let $a, y \in G$ be points of $G$ which are a distance $d$ apart and let $D=a \cdots x y$ be a path of length $d$ joining $a$ and $y$. Suppose

Received by the editors September 5, 1972.

AMS (MOS) subject classifications (1970). Primary 05 C99.

(c) American Mathematical Society 1974 
that $G^{*}=G-\{x, y\}$ is not a connected graph. Let $A$ be the component of $G^{*}$ that contains $a$. We note that since $D$ is a diameter of $G$, every point in $G^{*}-A$ is adjacent to $x$. Thus if $G^{*}-A$ contains a nontrivial component $B$, then for any $b, c \in B$ which are adjacent, every point in $G-\{b, c\}$ is joined to $x$ by a path, and hence $G-\{b, c\}$ is connected. Similarly, if there exists a point $e \in G^{*}-A$ which is adjacent to $y$, then $G-\{e, y\}$ is connected. Hence we may suppose that $G^{*}-A$ contains only isolated points and each of these points is adjacent only to $x$. Thus every point in $G^{*}-A$ is an endpoint of $G$ with joint $x$. Therefore since $G$ has no coincident endpoints, $G^{*}-A$ must consist of a single isolated point $f$. If $\dot{y}$ were an endpoint of $G$, then $f$ and $y$ would be coincident endpoints. Hence we may assume that $y$ is adjacent to some element of $A$, and so $G-\{f, x\}$ is connected.

THEOREM 1. If $G$ is a connected graph of even order, then $G^{2}$ has a 1-factor.

PROOF. Noting that the result holds for graphs of low order, we proceed by induction. Suppose that $G$ is connected of even order and that the theorem holds for graphs of smaller order. By Lemma 1 we may find points $x, y \in G$ such that $d(x, y) \leqq 2$ and $G-\{x, y\}$ is connected (for we may either choose $x$ and $y$ to be coincident endpoints or, if none such exist, we may take $x$ and $y$ to be the points guaranteed by Lemma 1). Thus $(G-\{x, y\})^{2}$ has a 1-factor, which together with the edge $x y$ of $G^{2}$ yields a 1 -factor for $G^{2}$.

Thus since the total graph $T(G)$ of a graph $G$ satisfies $T(G)=[S(G)]^{2}$ (Behzad [2]) where $S(G)$ is the subdivision graph of $G$, we have

COROLLARY 1. Every connected, total graph of even order has a 1-factor.

THEOREM 2. If $G$ is a connected graph of order $2 n(n>1)$ and $k$ is an integer, $1<k \leqq n$, such that every induced, connected subgraph of order $2 k$ has a 1-factor, then $G$ has a 1-factor.

Proof. We proceed by induction. The theorem is easily checked for $n=2,3$. Suppose that it holds for some $n-1(n \geqq 4)$. Let $G$ be a connected graph of order $2 n$ and suppose $k$ is such that $1<k \leqq n$ and every induced, connected subgraph of order $2 k$ contains a 1 -factor. Clearly we may assume that $k \leqq n-1$, the case $k=n$ being trivial. If $G$ contained two coincident endpoints, then we could extend the graph consisting of these two points and their common joint to a connected, induced subgraph of order $2 k$ which has no 1-factor. Hence $G$ does not contain two coincident endpoints and thus by Lemma 1 , there exist adjacent points $x, y \in G$ such that $G-\{x, y\}$ is connected. Thus by our inductive assumption, $G-\{x, y\}$ has a 1-factor which together with the edge $x y$ constitutes a 1-factor for $G$. 
COROLLARY 2. If $G$ is a connected graph of even order with no induced $K_{1,3}$, then $G$ has a 1 -factor.

ProOF. The only connected graph of order four which does not have a 1 -factor is $K_{1,3}$. Thus (except in the trivial case where $G$ is $K_{2}$ ) the corollary is the special case of the theorem with $k=2$.

Since no line graph can contain $K_{1,3}$ as an induced subgraph, we obtain

COROLLARY 3. Every connected line graph of even order has a 1-factor.

A 1-factor of a graph may be viewed as a partition of the points into two-element subsets in such a way that the elements of each set are adjacent. As a result of Corollary 3, we see that it is always possible to form a similar partition of the edges as long as there are an even number of them. Similarly, as a result of Corollary 1 , if a graph contains an even number of elements (i.e., points and lines), then we may partition the elements into subsets of order two so that the elements of each subset are either adjacent or incident.

As another immediate consequence of Corollary 2 we have

COROLlaRY 4. Every connected cubic graph in which every point lies in a triangle has a 1-factor.

REMARK. An alternate proof of Corollary 4 is obtained by noting that in any such graph other than $K_{4}$ the collection of those edges that lie in an even number of triangles constitutes a 1-factor. As an example, consider the graph in Figure 1. The edges which are numbered determine such a 1 -factor.

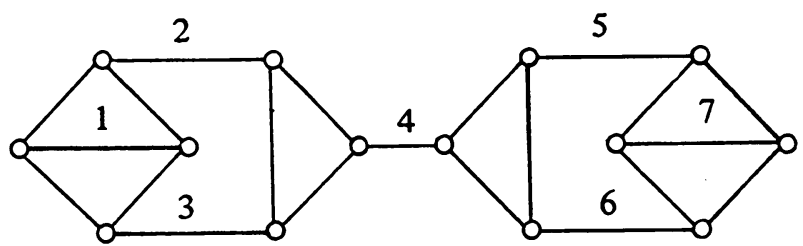

FIGURE 1

Our next result is an extension of Theorem 2. By an end-block we mean one which contains exactly one cutpoint.

THEOREM 3. Let $G$ be a connected graph of even order. If

(i) $G$ has exactly one block of even order, and

(ii) for each block $B$ of $G$, there exists an integer $k, 3<k \leqq|B|$ such that every induced, connected subgraph of $B$ having order $k$ contains $a$ 1-factor, then $G$ has a 1-factor. 
Proof. If $G$ has exactly one block, then $G$ has a 1 -factor by Theorem 2. Suppose $G$ is a graph satisfying the conditions (i) and (ii) such that the theorem holds for graphs with fewer blocks. Since $G$ has at least two endblocks, there exists an end-block $B$ of odd order. Let $b \in B$ be the unique cutpoint of $G$ lying in $B$, and let $k$ be the integer associated with $B$. Then $B-\{b\}$ is connected of even order and every induced, connected subgraph of $B-\{b\}$ having order $k$ has a 1 -factor (note $k \leqq|B|-1$ since $|B|$ is odd). Thus $B-\{b\}$ has a 1 -factor by Theorem 1. Also $(G-B) \cup\{b\}$ has fewer blocks than $G$ and satisfies (i) and (ii) and hence has a 1 -factor. Thus we obtain a 1 -factor for $G$.

The next theorem may be proven in a similar manner.

THEOREM 4. If $G$ is a connected graph of even order such that

(i) $G$ has exactly one block of even order, and

(ii) no block of $G$ contains an induced $K_{1,3}$,

then $G$ has a 1 -factor.

THEOREM 5. If $G$ is a connected graph of even order and every induced $K_{1,3}$ contains a bridge whose deletion results in two components of even order, then $G$ has a 1-factor.

PrOOF. We induct on the number of points in $G$ noting that the result holds for small orders. Let $G$ be a graph satisfying the hypotheses of the theorem and such that the theorem holds for graphs with fewer points than $G$. If $G$ has no induced $K_{1,3}$, then $G$ has a 1 -factor by Corollary 2 . If $G$ does not contain an induced $K_{1,3}$, let $e=a b$ be an edge of this $K_{1,3}$ such that the deletion of $e$ results in a graph having the two even components $A$ and $B$ with $a \in A$ and $b \in B$. Suppose $A$ contains an induced $K_{1,3}$. Let $f$ be an edge of this subgraph such that $G-\{f\}$ contains two components of even order. Since both endpoints of $f$ lie in $A, A-\{f\}$ consists of two components $C$ and $D$. We may assume $a \in D$. Then $C$ is one of the components of $G-\{f\}$ and hence has even order (as must $D$ also). Thus $A$ satisfies the conditions of the theorem and so must have a 1 -factor. Similarly, $B$ contains a 1 -factor which together with that for $A$ produces a 1 -factor for G.

Since this paper was submitted, we have learned that our Theorem 1, Corollary 1 , and Corollary 3 were discovered independently by $\mathbf{G}$. Chartrand, A. Polimeni, and J. Stewart and occur in their paper The existence of a 1-factor in line graphs, squares, and total graphs, which is to appear in Indagationes Mathematicae.

ACKNOWLEDGment. Thanks are due to Frank R. Bernhart for pointing out to me an error in a previous version of this paper. 


\section{REFERENCES}

1. L. W. Beineke and M. D. Plummer, On the 1-factors of a nonseparable graph, J. Combinatorial Theory 2 (1967), 285-289. MR 35 \#1499.

2. M. Behzad, A criterion for the planarity of the total graph of a graph, Proc. Cambridge Philos. Soc. 63 (1967), 679-681. MR 35 \#2771.

3. F. Harary, Graph theory, Addison-Wesley, Reading, Mass., 1969. MR 41 \#1566.

4. J. Petersen, Die Theorie der regulären Graphen, Acta Math. (1891), 193-220.

5. W. T. Tutte, The factorizations of linear graphs, J. London Math. Soc. 22 (1947), 107-111. MR 9, 297.

Department of Mathematics, University of South Carolina, Columbia, South Carolina 29208 